\title{
Thin accretion discs around neutron and quark stars
}

\author{
Z. Kovács ${ }^{1,2}$, K. S. Cheng ${ }^{3}$, and T. Harko ${ }^{3}$ \\ 1 Max-Planck-Institut für Radioastronomie, Auf dem Hügel 69, 53121 Bonn, Germany \\ e-mail: zkovacs@mpifr-bonn.mpg.de \\ ${ }^{2}$ Department of Experimental Physics, University of Szeged, Dóm Tér 9, Szeged 6720, Hungary \\ 3 Department of Physics and Center for Theoretical and Computational Physics, The University of Hong Kong, Hong Kong, \\ PR China \\ e-mail: [hrspksc; harko]@hkucc.hku.hk
}

Received 25 November 2008 / Accepted 18 March 2009

\section{ABSTRACT}

\begin{abstract}
Context. The possibility of observationally discriminating between various types of neutron stars, described by different equations of state of the nuclear matter, as well as differentiating neutron stars from other types of exotic objects, for example, quark stars, is one of the fundamental problems in contemporary astrophysics.

Aims. We consider and investigate carefully the possibility that different types of rapidly rotating neutron stars, as well as other type of compact general-relativistic objects, can be identified reliably by the study of the emission properties of the accretion discs around them.

Methods. We obtain the energy flux, temperature distribution, and emission spectrum from the accretion discs around several classes of rapidly rotating neutron stars, described by different equations of state for neutron matter, and for quark stars, described by the MIT bag model equation of state, and in the CFL (Color-Flavor-Locked) phase, respectively.

Results. Particular signatures appear in the electromagnetic spectrum, implying that the equation of state of the dense matter can be tested directly by using astrophysical observations of the emission spectra from accretion discs.
\end{abstract}

Key words. accretion, accretion disks - gravitation - starts: neutron - stars: rotation - stars: equation of state - dense matter

\section{Introduction}

The quark structure of the nucleon indicates that at high densities confined hadronic matter can undergo a phase transition to absolutely stable strange quark matter (Itoh 1970; Bodmer 1971; Witten 1984), a scenario that is referred to as the strange matter hypothesis. If the hypothesis is true, then some neutron stars should actually be stars made of strange quark matter (strange stars) (Alcock et al. 1986; Haensel et al. 1986). (For a general review of strange-star properties, see Cheng et al. 1998a)

There are several proposed mechanisms for forming quark stars. Quark stars are expected to form during the collapse of the core of a massive star, after the supernova explosion, and to be caused by a first or second order phase transition, that produces deconfined quark matter (Dai et al. 1995). The protoneutron-star core or the neutron-star core is a favorable environment for the conversion of ordinary matter to strange quark matter (Cheng et al. 1998b; Chan et al. 2009). Another possibility is that some neutron stars in low-mass X-ray binaries can accrete sufficient mass to undergo a phase transition and become strange stars (Cheng \& Dai 1996). This mechanism has been proposed as a source of radiation emission in cosmological $\gamma$-ray bursts (Cheng \& Dai 1998).

Based on numerical integration of the general-relativistic hydrostatic equilibrium equations, a complete description of the basic astrophysical properties (e.g. mass, radius, eccentricity, Keplerian frequency) of both static and rotating strange stars has been obtained, for different values of the bag constant and different equations of state of the strange star (Witten 1984; Haensel et al. 1986; Gondek-Rosinska et al. 2000;
Dey et al. 1998; Harko \& Cheng 2002). Rotational properties can discriminate between neutron and quark stars. Strange stars can have much shorter periods than neutron stars, on the order of $0.5 \mathrm{~ms}$ (Cheng et al. 1998a). The $r$-mode instabilities in rapidly rotating strange stars produce characteristic signatures in the evolution of pulsars with periods below $2.5 \mathrm{~ms}$, and some data on pulsar properties are consistent with this assumption (Madsen 2000). Strange stars could have a radius significantly less than that of neutron stars (Cheng et al. 1998a).

Photon emissivity is the basic parameter for determining macroscopic properties of stellar-type objects. Because of the very high plasma frequency $\omega_{\mathrm{p}}$ near the strange-matter edge, photon emissivity of strange matter is very low (Alcock et al. 1986). The spectrum of equilibrium photons is very hard, with $\hbar \omega>20 \mathrm{MeV}$. The problem of the soft-photon emissivity of quark matter at the surface of strange stars has also been considered (Cheng \& Harko 2003; Harko \& Cheng 2005). By taking into account the Landau-Pomeranchuk-Migdal effect and the absorption of the radiation in the external electron layer, the emissivity of the quark matter can be six orders of magnitude lower than the equilibrium black-body radiation.

The Coulomb barrier at the quark surface of a hot strange star may also be a powerful source of $\mathrm{e}^{+} \mathrm{e}^{-}$pairs, which are created in the extremely strong electric field of the barrier. At surface temperatures of around $10^{11} \mathrm{~K}$, the luminosity of the outflowing plasma may be on the order $\sim 10^{51} \mathrm{ergs}^{-1}$ (Usov 1998a,b; Harko \& Cheng 2006). Moreover, for about one day in the case of normal quark matter and up to a hundred years in the case of superconducting quark matter, the thermal luminosity at the star's surface, produced by photon emission 
and $\mathrm{e}^{+} \mathrm{e}^{-}$pair production, may be orders of magnitude higher than the Eddington limit (Page \& Usov 2002).

However, despite these very specific signatures for quark stars, a reliable method of discriminating them from neutron stars is still missing.

It is generally expected that most astrophysical objects grow substantially in mass via accretion. Accretion discs are flattened astronomical objects consisting of rapidly rotating gas that slowly spiral onto a central gravitating body, its gravitational energy being converted to heat. A fraction of the heat converted into radiation, which partially escapes, cooling down the accretion disc. The only information that we have about accretion-disc physics comes from this radiation, when it reaches radio, optical, and X-ray telescopes, allowing astronomers to analyze its electromagnetic spectrum, and its time variability. The efficient cooling by means of radiation over the disc surface prevents the disc from accumulating the heat generated by stresses and dynamical friction. In turn, the equilibrium subsequently achieved causes the disc to stabilize its thin vertical size. The thin disc has an inner edge at the marginally stable orbit of the compact-object potential, and the accreting plasma has a Keplerian motion in higher orbits (Page \& Thorne 1974; Thorne 1974).

Astrophysical observations have shown that around many compact objects, as well as around black-hole candidates, gas clouds surrounds both the central compact object, and an associated accretion disc, on a variety of scales ranging up to a tenth of a parsec or even a few hundred parsecs (Urry \& Padovani 1995). These clouds are assumed to form a geometrically and optically thick torus (or warped disc), which absorbs most of the ultraviolet radiation and the soft X-rays. The gas exists in either the molecular or atomic phase. Hence, important astrophysical information can be obtained by observing the motion of gas streams in the gravitational field of compact objects.

The first comprehensive theory of accretion discs was constructed in Shakura \& Sunyaev (1973) by using a Newtonian approach. This theory was extended to the general-relativistic models of mass accretion onto rotating black holes (Novikov \& Thorne 1973). These pioneering works developed thin steady-state accretion discs, where the accreting matter moves in Keplerian orbits. The hydrodynamical equilibrium in the disc is maintained by an efficient cooling mechanism via radiation transport. The photon flux emitted by the disc surface was studied for the assumption that the disc emits black-body radiation. The properties of radiant energy flux over the thin accretion discs were further analyzed, the effects of photon capture by the hole on the spin evolution also being taken into account (Page \& Thorne 1974; Thorne 1974). In these works, the efficiency with which black holes convert rest mass into outgoing radiation in the accretion process was also computed.

The disc and boundary layer luminosity for accreting rapidly rotating neutron stars with low magnetic fields in a fully general-relativistic manner were considered in Thampan \& Datta (1998). Sibgatullin \& Sunyaev (1998) analyzed the effect of the quadrupole component in the mass distribution of a rapidly rotating neutron star, on the energy release in the equatorial (or boundary) layer on the surface of the accreting star and in the accretion disc in the cases where the stellar radius is smaller (or larger) than the radius of the last stable circular orbit. The temperature profiles of (thin) accretion discs around rapidly rotating neutron stars (with low surface magnetic fields), taking into account the full effects of general relativity were obtained in Bhattacharyya et al. (2000). The calculations suggest that the neutron star in Cygnus X-2 rotates close to the centrifugal mass-shed limit. General-relativistic spectra from accretion discs around rotating neutron stars in the appropriate spacetime geometry for several different equations of state, spin rates, and masses of the compact object have been also computed (Bhattacharyya et al. 2001b). The spectra from the accretion discs around a rapidly rotating neutron star, taking into account the Doppler shift, the gravitational redshift, and the light-bending effects, were presented in Bhattacharyya et al. (2001c). Light bending significantly modifies the high-energy part of the spectrum. These results could be important for modeling the observed X-ray spectra of low-mass X-ray binaries, containing fast-spinning neutron stars. The temperature profiles of accretion discs around rapidly rotating strange stars, using constant gravitational mass equilibrium sequences of these objects, and considering the full effect of general relativity were computed in Bhattacharyya et al. (2001a). The structure parameters and temperature profiles obtained were compared with those of neutron stars, in an attempt to provide signatures for distinguishing between the two. The results obtained implied that it is possible to distinguish these objects from each other by sensitive observations acquired by future $\mathrm{X}$-ray detectors. The accretion-disc temperature profiles, disc luminosity, and boundary layer luminosity for rapidly rotating neutron stars, considering the full effect of general relativity, were calculated in Bhattacharyya (2002).

The emissivity properties of the accretion discs have been investigated for a wide range of compact objects, such as rotating and non-rotating boson or fermion stars (Torres 2002; Yuan et al. 2004; Guzman 2006), as well as for the modified $f(R)$ type theories of gravity (Pun et al. 2008a), for brane-world black-holes (Pun et al. 2008b), and for wormholes (Harko et al. 2008; Harko et al. 2009). The radiation power per unit area, the temperature of the disc, and the spectrum of the emitted radiation were given, and compared with similar properties for Schwarzschild or Kerr-Newman black holes of equal mass.

We consider a comparative systematic study of the properties of the thin accretion discs around both rapidly rotating neutron and strange stars, and obtain the basic physical parameters describing the disc, such as the emitted energy flux, the temperature distribution on the surface of the disc, as well as the spectrum of the emitted equilibrium black-body radiation.

To obtain the emissivity properties of the disc, the metric outside the rotating general-relativistic stars must be determined. In the present study, we calculate the equilibrium configurations of the rotating neutron and quark stars by using the RNS code, which was introduced in Stergioulas \& Friedman (1995), and discussed in detail in Stergioulas et al. (2003). This code was used for the study of different models of rotating neutron stars in Nozawa et al. (1998) and for the study of rapidly rotating strange stars (Stergioulas et al. 1999). The software provides the metric potentials for various types of compact rotating general-relativistic objects, which can be used to obtain the physical properties of the accretion discs. Particular signatures are apparent in the electromagnetic spectrum, which implies that it is possible to directly test the equation of state of dense matter by using astrophysical observations of the emission spectra from accretion discs.

The present paper is organized as follows. The properties of the general-relativistic thin accretion discs surrounding compact objects are briefly described in Sect. 2 . The equations of state used in the present study are presented in Sect. 3. In Sect. 4 we consider the radiation flux, spectrum, and efficiency of thin accretion discs around several classes of neutron stars and quark stars. We discuss our results and conclude in Sect. 5. 


\section{Thin accretion discs onto general relativistic compact objects}

A thin accretion disc is an accretion disc such that in cylindrical coordinates $(r, \phi, z)$ most of the matter lies close to the radial plane. The vertical size of a the thin accretion disc (defined along the $z$-axis) is negligible compared to its horizontal extension (defined along the radial direction $r$ ), i.e., the disc height $H$, equal to the maximum half thickness of the disc, is always far smaller than the characteristic radius $R$ of the disc, $H \ll R$ (Novikov \& Thorne 1973). The thin disc is in hydrodynamical equilibrium, and the pressure gradient and a vertical entropy gradient in the accreting matter are negligible. In the steady-state accretion-disc models, the mass-accretion rate $\dot{M}_{0}$ is supposed to be constant in time, and the physical quantities of the accreting matter are averaged over a characteristic time scale, e.g., $\Delta t$, and over the azimuthal angle $\Delta \phi=2 \pi$, for a total period of the orbits and for the height $H$. The plasma moves in Keplerian orbits around the compact object, with a rotational velocity $\Omega$. The plasma particles have a specific energy $\widetilde{E}$, and a specific angular momentum $\widetilde{L}$, which depend only on the radii of the orbits. The four-velocity of the particles is $u^{\mu}$, and the disc has an averaged surface density $\Sigma$. The accreted matter is modeled by an anisotropic fluid source, where the density $\rho_{0}$ (the specific heat is neglected), the energy flow vector $q^{\mu}$, and the stress tensor $t^{\mu v}$ are measured in an averaged rest-frame. The energy-momentum tensor takes the form (Novikov \& Thorne 1973)

$T^{\mu v}=\rho_{0} u^{\mu} u^{v}+2 u^{(\mu} q^{v)}+t^{\mu \nu}$,

where $u_{\mu} q^{\mu}=0, u_{\mu} t^{\mu v}=0$. The four-vectors of the energy and of the angular momentum flux are defined by $-E^{\mu} \equiv T^{\mu}{ }_{v}(\partial / \partial t)^{v}$ and $J^{\mu} \equiv T^{\mu}{ }_{v}(\partial / \partial \phi)^{v}$, respectively. The four-dimensional conservation laws of the rest mass, energy, and angular momentum of the plasma represent the structure equations of the thin disc. By integrating the equation of the rest-mass conservation, $\nabla_{\mu}\left(\rho_{0} u^{\mu}\right)=0$, it follows that the time-averaged accretion-rate $\dot{M}_{0}$ is independent of the disc radius:

$\dot{M}_{0} \equiv-2 \pi r \sum u^{r}=$ const.,

where a dot represents the derivative with respect to the time coordinate (Page \& Thorne 1974). The average rest-mass density is defined by $\Sigma(r)=\int_{-H}^{H}\left\langle\rho_{0}\right\rangle \mathrm{d} z$, where $\left\langle\rho_{0}\right\rangle$ is the rest-mass density averaged over $\Delta t$ and $2 \pi$. The conservation law $\nabla_{\mu} E^{\mu}=0$ of the energy can be written in an integral form as

$\left[\dot{M}_{0} \widetilde{E}-2 \pi r \Omega W_{\phi}^{r}\right]_{, r}=4 \pi \sqrt{-g} F \widetilde{E}$,

where a comma denotes the derivative with respect to the radial coordinate $r$. Equation (3) shows the balance between the energy transported by the rest-mass flow, the dynamical stresses in the disc, and the energy radiated away from the surface of the disc, respectively. The torque $W_{\phi}{ }^{r}$ in Eq. (3) is given by

$W_{\phi}^{r}=\int_{-H}^{H}\left\langle t_{\phi}^{r}\right\rangle \mathrm{d} z$

where $\left\langle t_{\phi}{ }^{r}\right\rangle$ is the $\phi-r$ component of the stress tensor, averaged over $\Delta t$ and over a $2 \pi$ angle. The law of the angular-momentum conservation, $\nabla_{\mu} J^{\mu}=0$, states in its integral form, the balance of the three forms of angular momentum transport,

$\left[\dot{M}_{0} \widetilde{L}-2 \pi r W_{\phi}^{r}\right]_{, r}=4 \pi \sqrt{-g} F \widetilde{L}$.
By eliminating $W_{\phi}{ }^{r}$ from Eqs. (3) and (5), and applying the universal energy-angular momentum relation $\mathrm{d} E=\Omega \mathrm{d} J$ for circular geodesic orbits in the form $\widetilde{E}_{, r}=\Omega \widetilde{L}_{r}$, the flux of the radiant energy over the disc can be expressed in terms of the specific energy, angular momentum, and the angular velocity of the black hole. The flux integral then provides an expression for the energy flux $F(r)$, which is given by (Page \& Thorne 1974; Thorne 1974)

$F(r)=-\frac{\dot{M}_{0}}{4 \pi \sqrt{-g}} \frac{\Omega_{, r}}{(\widetilde{E}-\Omega \widetilde{L})^{2}} \int_{r_{\mathrm{ms}}}^{r}(\widetilde{E}-\Omega \widetilde{L}) \widetilde{L}_{, r} \mathrm{~d} r$

where the no-torque inner boundary conditions were also prescribed (Page \& Thorne 1974). This means that the torque vanishes at the inner edge of the disc, since the matter at the marginally stable orbit $r_{\mathrm{ms}}$ falls freely onto the black hole, and cannot exert considerable torque on the disc. The latter assumption is valid as long as strong magnetic fields do not exist in the plunging region, where matter falls into the hole.

Once the geometry of the space-time is known, we can derive the time-averaged radial distribution of photon emission for accretion discs around black holes, and determine the efficiency of the conversion of the rest mass into outgoing radiation. After obtaining the radial dependence of the angular velocity $\Omega$, the specific energy $\widetilde{E}$, and the specific angular momentum $\widetilde{L}$ of the particles moving on circular orbits around the black holes, respectively, we can compute the flux integral given by Eq. (6).

We consider an arbitrary stationary and axially symmetric geometry,

$\mathrm{d} s^{2}=g_{t t} \mathrm{~d} t^{2}+g_{t \phi} \mathrm{d} t \mathrm{~d} \phi+g_{r r} \mathrm{~d} r^{2}+g_{\theta \theta} \mathrm{d} \theta^{2}+g_{\phi \phi} \mathrm{d} \phi^{2}$,

where in the equatorial approximation $(|\theta-\pi / 2| \ll 1)$, and the metric functions $g_{t t}, g_{t \phi}, g_{r}, g_{\theta \theta}$, and $g_{\phi \phi}$ depend only on the radial coordinate $r$. The geodesic equations take the form

$\frac{\mathrm{d} t}{\mathrm{~d} \tau}=\frac{\widetilde{E} g_{\phi \phi}+\widetilde{L} g_{t \phi}}{g_{t \phi}^{2}-g_{t t} g_{\phi \phi}}, \quad \frac{\mathrm{d} \phi}{\mathrm{d} \tau}=-\frac{\widetilde{E} g_{t \phi}+\widetilde{L} g_{t t}}{g_{t \phi}^{2}-g_{t t} g_{\phi \phi}}$,

and

$g_{r r}\left(\frac{\mathrm{d} r}{\mathrm{~d} \tau}\right)^{2}=V(r)$

respectively, where $\tau$ is the affine parameter, and the potential term $V(r)$ is defined by

$V(r) \equiv-1+\frac{\widetilde{E} g_{\phi \phi}+2 \widetilde{E L} g_{t \phi}+\widetilde{L}^{2} g_{t t}}{g_{t \phi}^{2}-g_{t t} g_{\phi \phi}}$

For circular orbits in the equatorial plane, the conditions $V(r)=$ 0 and $V_{, r}(r)=0$ must hold for all disc configurations. From these conditions, one can obtain the specific energy $\widetilde{E}$, the specific angular-momentum $\widetilde{L}$ and the angular velocity $\Omega$ of the particles moving on circular orbits around spinning, general-relativistic stars as

$\widetilde{E}=-\frac{g_{t t}+g_{t \phi} \Omega}{\sqrt{-g_{t t}-2 g_{t \phi} \Omega-g_{\phi \phi} \Omega^{2}}}$,

$\widetilde{L}=\frac{g_{t \phi}+g_{\phi \phi} \Omega}{\sqrt{-g_{t t}-2 g_{t \phi} \Omega-g_{\phi \phi} \Omega^{2}}}$,

$\Omega=\frac{\mathrm{d} \phi}{\mathrm{d} t}=\frac{-g_{t \phi, r}+\sqrt{\left(g_{t \phi, r}\right)^{2}-g_{t t, r} g_{\phi \phi, r}}}{g_{\phi \phi, r}}$. 
The marginally stable orbits $r_{\mathrm{ms}}$ around the central object are determined by the condition $\left.V_{, r r}(r)\right|_{r=r_{\mathrm{ms}}}=0$, which is equivalent to the equation

$$
\left[\widetilde{E}^{2} g_{\phi \phi, r r}+2 \widetilde{E L} g_{t \phi, r r}+\widetilde{L}^{2} g_{t t, r r}-\left(g_{t \phi}^{2}-g_{t t} g_{\phi \phi}\right)_{, r r}\right]_{r=r_{\mathrm{ms}}}=0
$$

By inserting Eqs. (11), (12) into Eq. (14), and solving the resulting equation for $r_{\mathrm{ms}}$, we obtain marginally stable orbits, once the metric coefficients $g_{t t}, g_{t \phi}$ and $g_{\phi \phi}$ are explicitly given.

The accreting matter in the steady-state thin-disc model is supposed to be in thermodynamical equilibrium. Therefore, the radiation emitted by the disc surface can be considered to be a perfect black-body radiation, where the energy flux is given by $F(r)=\sigma T^{4}(r)(\sigma$ is the Stefan-Boltzmann constant), and the observed luminosity $L(v)$ has a redshifted black-body spectrum, given by (Torres 2002):

$L(v)=4 \pi d^{2} I(v)=\frac{8}{\pi} \cos \gamma \int_{r_{i}}^{r_{f}} \int_{0}^{2 \pi} \frac{v_{\mathrm{e}}^{3} r \mathrm{~d} \phi \mathrm{d} r}{\exp \left(v_{\mathrm{e}} / T\right)-1}$.

Here $d$ is the distance to the source, $I(v)$ is the Planck distribution function, $\gamma$ is the disc inclination angle, and $r_{\mathrm{i}}$ and $r_{\mathrm{f}}$ indicate the position of the inner and outer edge of the disc, respectively. We assume that $r_{\mathrm{i}}=r_{\mathrm{ms}}$ and $r_{\mathrm{f}} \rightarrow \infty$, since we expect the flux over the disc surface to vanish at $r \rightarrow \infty$ for any kind of general-relativistic compact-object geometry. The emitted frequency is given by $v_{\mathrm{e}}=v(1+z)$, where the redshift factor can be written as

$1+z=\frac{1+\Omega r \sin \phi \sin \gamma}{\sqrt{-g_{t t}-2 \Omega g_{t \phi}-\Omega^{2} g_{\phi \phi}}}$

where we have neglected light bending (Luminet 1979; Bhattacharyya et al. 2001b).

The flux and emission spectra of accretion discs around compact objects satisfy simple scaling relations, related to the scaling transformation of the radial coordinate, given by $r \rightarrow \widetilde{r}=r / M$, where $M$ is the mass of the black hole. Generally, the metric tensor coefficients are invariant with respect to this transformation, while the specific energy, the angular momentum, and the angular velocity transform as $\widetilde{E} \rightarrow \widetilde{E}, \widetilde{L} \rightarrow M \widetilde{L}$, and $\Omega \rightarrow \widetilde{\Omega} / M$, respectively. The flux scales as $F(r) \rightarrow F(r) / M^{4}$, giving the simple transformation law for temperature of $T(r) \rightarrow T(r) / M$. By also rescaling the frequency of the emitted radiation by $v \rightarrow \widetilde{v}=$ $v / M$, the luminosity of the disc is given by $L(v) \rightarrow L \widetilde{v}) / M$. On the other hand, the flux is proportional to the accretion rate $\dot{M}_{0}$, and therefore an increase in the accretion rate leads to a linear increase in the radiation emission flux from the disc.

The efficiency $\epsilon$ with which the central object converts rest mass into outgoing radiation is the other important physical parameter characterizing the properties of the accretion discs. The efficiency is defined by the ratio of two rates measured at infinity: the rate of the radiation corresponding to the energy of photons escaping from the disc surface to infinity, and the rate at which mass-energy is transported to the compact object. If all the emitted photons can escape to infinity, the efficiency depends only on the specific energy measured in the marginally stable orbit $r_{\mathrm{ms}}$,

$\epsilon=1-\left.\widetilde{E}\right|_{r=r_{\mathrm{ms}}}$.

There are some solutions for the geometry of neutron and quark stars where $r_{\mathrm{ms}}$ corresponds to the surface of the star or is even smaller than the surface radius of the central object. In this case, the inner edge of the disc touches the surface of the star, and plasma influenced by any perturbation caused by hydro- or magnetohydrodynamic instabilities in the disc will leave the disc and hit the surface. The energy $\widetilde{E}_{\mathrm{e}}$ transferred to the star from the disc is then measured at the radius $R_{\mathrm{e}}$ of the star, and the efficiency takes the form

$\epsilon=1-\widetilde{E}_{\mathrm{e}}$

where $\widetilde{E}_{\mathrm{e}}=\left.\widetilde{E}\right|_{r=R_{\mathrm{e}}}$. For Schwarzschild black holes, the efficiency is about $6 \%$, regardless of whether photon capture by the black hole is considered. Ignoring the capture of radiation by the black hole, $\epsilon$ is found to be $42 \%$ for rapidly rotating black holes, whereas the efficiency is $40 \%$ for photon capture in the Kerr potential. For neutron and quark stars, the efficiency varies across broader range, especially if we account for $\widetilde{E}_{\mathrm{ms}}$ and $\widetilde{E}_{\mathrm{e}}$ having very different values in different neutron and quark star models.

\section{Equations of state}

To obtain a consistent and realistic physical description of rotating general-relativistic neutron and quark stars, first we must adopt the equations of state for the dense neutron and quark matter, respectively. In the present study of accretion discs around rapidly rotating neutron and quark stars, we consider the following equations of state:

1) Akmal-Pandharipande-Ravenhall (APR) EOS (Akmal et al. 1998). EOS APR has been obtained by using the variational chain summation methods and the Argonne $v_{18}$ twonucleon interaction. Boost corrections to the two-nucleon interaction, which provide the leading relativistic effect of order $(v / c)^{2}$, as well as three-nucleon interactions, are also included in the nuclear Hamiltonian. The density range is from $2 \times 10^{14} \mathrm{~g} / \mathrm{cm}^{3}$ to $2.6 \times 10^{15} \mathrm{~g} / \mathrm{cm}^{3}$. The maximum mass limit in the static case for this EOS is $2.20 M_{\odot}$. We join this equation of state to the composite $\operatorname{BBP}\left(\epsilon / c^{2}>4.3 \times\right.$ $\left.10^{11} \mathrm{~g} / \mathrm{cm}^{3}\right)$ (Baym et al. 1971a) - BPS $\left(10^{4} \mathrm{~g} / \mathrm{cm}^{3}<4.3 \times\right.$ $\left.10^{11} \mathrm{~g} / \mathrm{cm}^{3}\right)$ (Baym et al. 1971b) - FMT $\left(\epsilon / c^{2}<10^{4} \mathrm{~g} / \mathrm{cm}^{3}\right)$ (Feynman et al. 1949) equations of state.

2) Douchin-Haensel (DH) EOS (Douchin \& Haensel 2001). EOS DH is an equation of state for neutron-star matter, that describes both the neutron star crust and the liquid core. It is based on the effective nuclear interaction SLy of the Skyrme type, which is particularly suited for calculating of the properties of very neutron-rich matter. The structure of the crust, and its EOS, is calculated for zero-temperature approximation, and the assumption of ground state composition. The EOS of the liquid core is calculated by assuming a (minimal) пре $\mu$ composition. The density ranges from $3.49 \times 10^{11} \mathrm{~g} / \mathrm{cm}^{3}$ to $4.04 \times 10^{15} \mathrm{~g} / \mathrm{cm}^{3}$. The minimum and maximum masses of the static neutron stars for this EOS are $0.094 M_{\odot}$ and $2.05 M_{\odot}$, respectively.

3) Shen-Toki-Oyamatsu-Sumiyoshi (STOS)EOS (Shen et al. 1998). The STOS equation of state of nuclear matter is obtained by using the relativistic mean-field theory with nonlinear $\sigma$ and $\omega$ terms for a wide range of density and temperature, and various proton fractions. The EOS was specifically designed for supernova simulations and for neutron star calculations. The Thomas-Fermi approximation is used to describe inhomogeneous matter, where heavy nuclei are formed together with free nucleon gas. We consider the STOS EOS for several temperatures, namely $T=0, T=0.5$ and $T=1 \mathrm{MeV}$, respectively. The temperature is mentioned for each STOS equation of state, so that, for example, STOS 
0 represents the STOS EOS for $T=0$. For the proton fraction we chose the value $Y_{\mathrm{p}}=0.001$ in order to avoid the negative pressure regime for low baryon mass densities.

4) Relativistic Mean Field (RMF) equations of state with isovector scalar mean field corresponding to the $\delta$-meson-RMF soft and RMF stiff EOS (Kubis \& Kutschera 1997). While the $\delta$-meson mean field vanishes in symmetric nuclear matter, it can influence the properties of asymmetric nuclear matter in neutron stars. The relativistic mean-field contribution by the $\delta$-field to the nuclear symmetry energy is negative. The energy per particle of neutron matter is then higher at high densities than the energy with no $\delta$-field included. The proton fraction of $\beta$-stable matter also increases. The splitting of proton and neutron effective masses by the $\delta$-field can affect transport properties of neutron-star matter. The equations of state can be parameterized by the coupling parameters $C_{\sigma}^{2}=g_{\sigma}^{2} / m_{\sigma}^{2}, C_{\omega}^{2}=g_{\omega}^{2} / m_{\omega}^{2}, \bar{b}=b / g_{\sigma}^{3}$, and $\bar{c}=c / g_{\sigma}^{4}$, where $m_{\sigma}$ and $m_{\omega}$ are the masses of the respective mesons, and $b$ and $c$ are the coefficients in the potential energy $U(\sigma)$ of the $\sigma$-field. The soft RMF EOS is parameterized by $C_{\sigma}^{2}=1.582 \mathrm{fm}^{2}, C_{\omega}^{2}=1.019 \mathrm{fm}^{2}, \bar{b}=-0.7188$ and $\bar{c}=6.563$, while the stiff RMF EOS is parameterized by $C_{\sigma}^{2}=11.25 \mathrm{fm}^{2}, C_{\omega}^{2}=6.483 \mathrm{fm}^{2}, \bar{b}=0.003825$ and $\bar{c}=3.5 \times 10^{-6}$, respectively.

5) Baldo-Bombaci-Burgio (BBB) EOS (Baldo et al. 1997). The BBB EOS is an EOS for asymmetric nuclear matter, derived from the Brueckner-Bethe-Goldstone many-body theory with explicit three-body forces. Two EOS's are obtained, one corresponding to the Argonne AV14 (BBBAV14), and the other to the Paris two-body nuclear force (BBBParis), implemented by the Urbana model for the three-body force. The maximum static mass configurations are $M_{\max }=1.8 M_{\odot}$ and $M_{\max }=1.94 M_{\odot}$ when the AV14 and Paris interactions are used, respectively. The onset of direct Urca processes occurs at densities of $n \geq 0.65 \mathrm{fm}^{-3}$ for the AV14 potential and $n \geq 0.54 \mathrm{fm}^{-3}$ for the Paris potential. A comparison with other microscopic models for the EOS shows noticeable differences. The density ranges from $1.35 \times 10^{14} \mathrm{~g} / \mathrm{cm}^{3}$ to $3.507 \times 10^{15} \mathrm{~g} / \mathrm{cm}^{3}$.

6) Bag model equation of state (Q) EOS (Witten 1984; Cheng et al. 1998a). For describing the quark matter, we adopt first a simple phenomenological description, based on the MIT bag model equation of state, in which the pressure $p$ is related to the energy density $\rho$ by

$p=\frac{1}{3}(\rho-4 B)$,

where $B$ is the difference between the energy density of the perturbative and non-perturbative QCD vacuum (the bag constant), of value $B=4.2 \times 10^{14} \mathrm{~g} / \mathrm{cm}^{3}$.

7) Color-Flavor-Locked (CFL) EOS. It is generally agreed that the color-flavor-locked (CFL) state is likely to be the ground state of matter, at least for asymptotic densities, and even if the quark masses are unequal (Alford et al. 1999; Rapp et al. 2000; Alford et al. 2007). Moreover, the equal number of flavors is enforced by symmetry, and electrons are absent, since the mixture is automatically neutral. By assuming that the mass $m_{s}$ of the $s$ quark is not high compared to the chemical potential $\mu$, the thermodynamical potential of the quark matter in CFL phase can be approximated as (Lugones \& Horvath 2002)

$\Omega_{\mathrm{CFL}}=-\frac{3 \mu^{4}}{4 \pi^{2}}+\frac{3 m_{s}^{2}}{4 \pi^{2}}-\frac{1-12 \ln \left(m_{s} / 2 \mu\right)}{32 \pi^{2}} m_{s}^{4}-\frac{3}{\pi^{2}} \Delta^{2} \mu^{2}+B,($

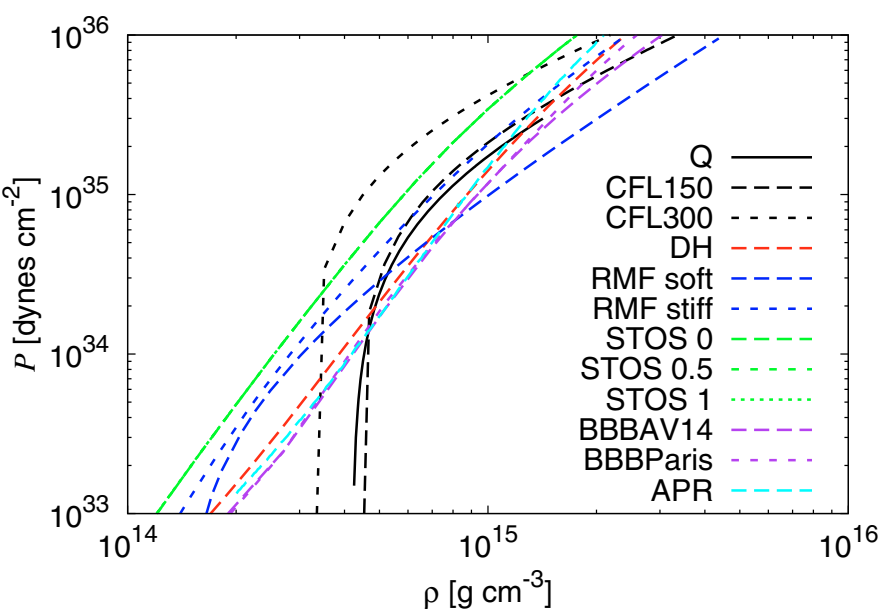

Fig. 1. Pressure as a function of density (in a logarithmic scale) for the equations of state Q, CFL150, CFL300, DH, RMF soft, RMF stiff, STOS 0. STOS 0.5, STOS 1, BBBAV14, BBBParis, and APR, respectively.

where $\Delta$ is the gap energy. By using this expression the pressure $P$ of the quark matter in the CFL phase can be obtained as an explicit function of the energy density $\varepsilon$ in the form (Lugones \& Horvath 2002)

$P=\frac{1}{3}(\varepsilon-4 B)-\frac{2 \Delta^{2} \delta^{2}}{\pi^{2}}+\frac{m_{s}^{2} \delta^{2}}{2 \pi^{2}}$,

where

$\delta^{2}=-\alpha+\sqrt{\alpha^{2}+\frac{4}{9} \pi^{2}(\varepsilon-B)}$,

and $\alpha=-m_{s}^{2} / 6+2 \Delta^{2} / 3$. In the following, the value of the gap energy $\Delta$ considered in each case is also mentioned for the CFL equation of state, so that, for example, CFL150 represents the CFL EOS for $\Delta=150 \mathrm{MeV}$. For the mass of the strange quark, we adopt the value $m_{s}=150 \mathrm{MeV}$. The maximum mass of the stars in the CFL model is given by $M_{\max }=1.96 M_{\odot}(1+\delta) / \sqrt{B_{60}}$, where $\delta=0.15\left(\Delta / 100 \mathrm{MeV}^{2}\left(60 \mathrm{MeV} \mathrm{fm}^{-3} / B\right)\right.$ (Horvath \& Lugones 2004). Hence, high maximum masses $\left(M>4 M_{\odot}\right)$ can be found for standard values of $B$ and $\Delta \geq 250 \mathrm{MeV}$. For a discussion of the maximum mass of both rotating and non-rotating general-relativistic objects, we refer the reader to Stergioulas et al. (2003). For rotating stars, the maximum mass can be of the order of $M_{\max } \approx$ $6.1 M_{\odot}\left(2 \times 10^{14} \mathrm{~g} \mathrm{~cm}^{-3} / \rho_{\mathrm{m}}\right)^{1 / 2}$, where $\rho_{\mathrm{m}}$ is the matching density.

The pressure-density relation is presented for the considered equations of state in Fig. 1.

\section{Thin disc accretion onto neutron and quark stars}

We consider the properties of the general-relativistic thin accretion discs formed around neutron stars and quark stars. The metric outside the rotating compact general-relativistic stars can be described, in quasi-isotropic coordinates, as

$\mathrm{d} s^{2}=-\mathrm{e}^{\bar{\gamma}+\bar{\rho}} \mathrm{d} t^{2}+\mathrm{e}^{2 \bar{\alpha}}\left(\mathrm{d} \bar{r}^{2}+\bar{r}^{2} \mathrm{~d} \theta^{2}\right)+\mathrm{e}^{\bar{\gamma}-\bar{\rho}} \bar{r}^{2} \sin ^{2} \theta(\mathrm{d} \phi-\bar{\omega} \mathrm{d} t)^{2}$,

where the metric potentials $\bar{\gamma}, \bar{\rho}, \bar{\alpha}$, and the angular velocity of the stellar fluid relative to the local inertial frame $\bar{\omega}$ are all 
Table 1. Physical parameters of the compact stars with total mass $M \approx 1.8 M_{\odot}$ and rotational frequency $\Omega \approx 5 \times 10^{3} \mathrm{~s}^{-1}$.

\begin{tabular}{llllllllllll}
\hline \hline EOS & DH & RMF soft RMF stiff & STOS 0 & STOS 0.5 & STOS 1 & BBBAV14 & BBBParis & APR & $Q$ & CFL150 \\
\hline$\rho_{\mathrm{c}}\left[10^{15} \mathrm{~g} / \mathrm{cm}^{3}\right]$ & 1.29 & 2.00 & 0.57 & 0.369 & 0.383 & 0.40 & 2.15 & 1.70 & 1.225 & 0.931 & 0.71 \\
$M\left[M_{\odot}\right]$ & 1.81 & 1.52 & 1.80 & 1.85 & 1.80 & 1.79 & 1.80 & 1.80 & 1.80 & 1.79 & 1.80 \\
$M_{0}\left[M_{\odot}\right]$ & 2.05 & 1.70 & 2.00 & 2.01 & 1.95 & 1.93 & 2.08 & 2.07 & 2.11 & 2.09 & 2.09 \\
$R_{\mathrm{e}}[\mathrm{km}]$ & 12.01 & 11.34 & 15.79 & 21.03 & 22.84 & 22.72 & 10.57 & 10.98 & 10.99 & 11.79 & 12.36 \\
$\Omega\left[10^{3} \mathrm{~s}^{-1}\right]$ & 4.99 & 5.00 & 5.00 & 4.90 & 4.71 & 4.45 & 5.01 & 5.00 & 5.00 & 4.79 & 5.00 \\
$\Omega_{\mathrm{p}}\left[10^{3} \mathrm{~s}^{-1}\right]$ & 11.16 & 11.67 & 7.97 & 5.37 & 4.56 & 4.54 & 13.96 & 13.19 & 13.23 & 11.97 & 11.28 \\
$T / W\left[10^{-2}\right]$ & 3.43 & 3.31 & 8.93 & 14.91 & 12.25 & 9.83 & 2.35 & 2.66 & 3.41 & 4.45 & 5.64 \\
$c J / G M_{\odot}^{2}$ & 1.15 & 0.82 & 2.00 & 2.95 & 2.49 & 2.15 & 0.95 & 1.00 & 1.12 & 1.28 & 1.50 \\
$I\left[10^{45} \mathrm{~g} \mathrm{~cm}^{2}\right]$ & 2.03 & 1.45 & 3.52 & 5.30 & 4.65 & 4.25 & 1.66 & 1.76 & 1.98 & 2.35 & 2.63 \\
$\Phi_{2}\left[10^{43} \mathrm{~g} \mathrm{~cm}^{2}\right]$ & 8.54 & 6.73 & 43.82 & 106.53 & 80.44 & 61.22 & 4.42 & 5.43 & 7.87 & 1.30 & 1.91 \\
$h_{+}[\mathrm{km}]$ & 6.85 & 0.00 & 0.00 & 0.00 & 0.00 & 0.00 & 3.19 & 2.69 & 0.00 & 0.00 & 0.00 \\
$h_{-}[\mathrm{km}]$ & 7.48 & -2.35 & -3.40 & 7.91 & 3.87 & 2.27 & 8.14 & 7.90 & -2.11 & 0.00 & 0.00 \\
$\omega_{\mathrm{c}} / \Omega\left[10^{-1}\right]$ & 5.85 & 5.52 & 4.52 & 4.10 & 4.07 & 4.08 & 6.67 & 6.34 & 5.86 & 5.27 & 5.00 \\
$r_{\mathrm{e}}[\mathrm{km}]$ & 9.10 & 8.90 & 12.85 & 18.00 & 19.97 & 19.91 & 7.64 & 8.06 & 8.05 & 8.86 & 9.40 \\
$r_{\mathrm{p}} / r_{\mathrm{e}}$ & 0.88 & 0.88 & 0.72 & 0.54 & 0.53 & 0.58 & 0.92 & 0.91 & 0.90 & 0.87 & 0.84 \\
\hline
\end{tabular}

functions of the quasi-isotropic radial coordinate $\bar{r}$ and the polar angle $\theta$. The RNS code produces the metric functions in a quasi-spheroidal coordinate system, as functions of the parameter $s=\bar{r} /\left(\bar{r}+\bar{r}_{\mathrm{e}}\right)$, where $\bar{r}_{\mathrm{e}}$ is the equatorial radius of the star, which we have converted into Schwarzschild-type coordinates $r$ according to the equation $r=\bar{r} \exp [(\bar{\gamma}-\bar{\rho}) / 2]$. To obtain the radius of the marginally (or innermost) stable circular orbits $r_{\mathrm{ms}}$, we have used a truncated form of the analytical approximation given as (Shibata \& Sasaki 1998),

$$
\begin{aligned}
\frac{r_{\mathrm{ms}}}{6 M} \approx & \left(1-0.54433 q-0.22619 q^{2}+0.17989 Q_{2}-0.23002 q^{2}\right. \\
& \left.+0.26296 q Q_{2}-0.29693 q^{4}+0.44546 q^{2} Q_{2}\right)
\end{aligned}
$$

where $q=J / M^{2}, Q_{2}=-M_{2} / M^{3}, J$ is the spin angular momentum, and $M_{2}$ is the quadrupole moment. Once the metric outside the rotating stars is known, from Eqs. (12), (13), we obtain the angular velocity, the specific energy, and the specific angular momentum of the orbiting plasma particles in the disc. We then calculate the integral given by Eq. (6), measuring the photon flux emitted by the disc surface in thermodynamical equilibrium. From the flux, we obtain the temperature distribution of the disc, as well as the spectra of the emitted radiation. For all our calculations, we use an accretion rate of $\dot{M}=1 \times 10^{-12} M_{\odot} / \mathrm{yr}$, and assume that $\cos \gamma=1$. To compare the thermal and emission properties of the thin accretion discs onto neutron and quark stars, we consider several classes of models in which both neutron and quark stars have some common characteristics.

\subsection{Accretion discs around neutron and quark stars of similar mass and angular velocity}

As a first example of the electromagnetic radiation characteristics of accretion discs, we consider the properties of discs around rapidly rotating neutron and quark stars of the same gravitational mass $M$ and angular velocity $\Omega$. If $M$ and $\Omega$ as well as the electromagnetic characteristics of the radiation emission from the disc could be determined by using astronomical and astrophysical observations, one may attempt to identify the type of central star by analyzing the differences in the physical parameters (flux, temperature, and spectrum) of the disc. The physical parameters of the accretion discs are obtained for a fixed total mass

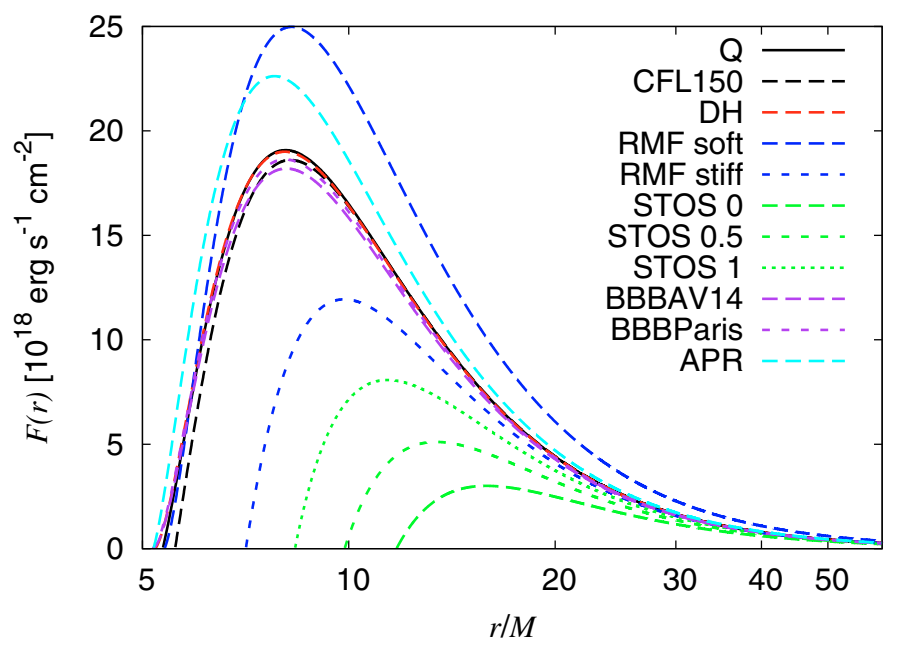

Fig. 2. The time-averaged flux radiated by a thin accretion disc around rotating neutron and quark stars, with the same total mass $M=1.8 M_{\odot}$, and rotational velocity $\Omega=5 \times 10^{3} \mathrm{~s}^{-1}$.

and rotational frequency for all the equations of state discussed in Sect. 3.

In Table 1, we present the main physical parameters of the considered neutron and quark stars, of total mass equal to values around $1.8 M_{\odot}$, and the angular velocity fixed to values around $5 \times 10^{3} \mathrm{~s}^{-1}$. In this, and the following tables, $\rho_{\mathrm{c}}$ is the central density, $M$ is the gravitational mass, $M_{0}$ is the rest mass, $R_{\mathrm{e}}$ is the circumferential radius at the equator, $\Omega$ is the angular velocity, $\Omega_{\mathrm{p}}$ is the angular velocity of a particle in circular orbit at the equator, $T / W$ is the rotational-gravitational energy ratio, $c J / G M_{\odot}^{2}$ is the angular momentum, $I$ is the moment of inertia, $\Phi_{2}$ gives the mass quadrupole moment $M_{2}$ so that $M_{2}=c^{4} \Phi_{2} /\left(G^{2} M^{3} M_{\odot}^{3}\right), h_{+}$ is the height from the surface of the last stable co-rotating circular orbit in the equatorial plane, $h_{-}$is the height from surface of the last stable counter-rotating circular orbit in the equatorial plane, $\omega_{\mathrm{c}} / \Omega$ is the ratio of the central value of the potential $\omega$ to $\Omega, r_{\mathrm{e}}$ is the coordinate equatorial radius, and $r_{\mathrm{p}} / r_{\mathrm{e}}$ is the axes ratio (polar to equatorial), respectively.

In Fig. 2, we present the total flux $F(r)$ radiated by the accretion disc around the stellar models DH, RMF soft/stiff, STOS $T=0.0,0.5$, and 1.0, BBBAV14, BBBParis, APR, Q, and CFL150, respectively, all the stars having the mass of $1.8 M_{\odot}$ 


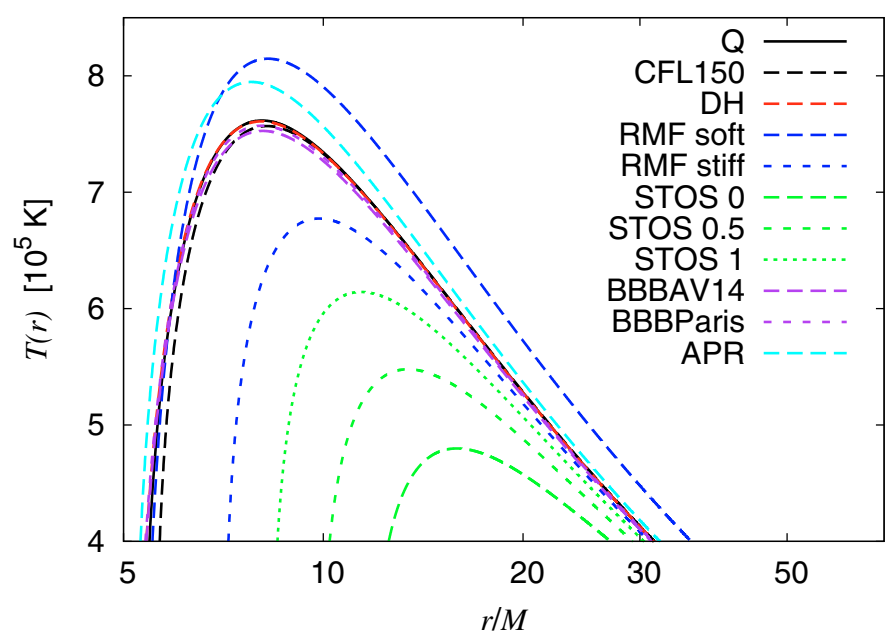

Fig. 3. The temperature distribution in a thin accretion disc around rotating neutron and quark stars with the same total mass $M=1.8 M_{\odot}$, and rotational velocity $\Omega=5 \times 10^{3} \mathrm{~s}^{-1}$.

and the angular velocity $5 \times 10^{3} \mathrm{~s}^{-1}$. We can distinguish two types of behavior from the curves in this figure.

The first group contains models with relatively small stellar radii, on the order of $10-12 \mathrm{~km}$. The stars with EOS APR, BBBAV14, BBBParis, and DH belong to this group, and the inner edges of the accretion disc (indicated by the left boundary of the radial flux profile) are located at low dimensionless radii, close to each other, with $r / M \simeq 5.2-5.6$. The shape of their flux profiles is rather similar, and only the stellar model APR produces a maximal flux value higher by a factor of 1.4 than the others. The maximal flux values of stars with the $\mathrm{DH}$ and BBBParis type EOS's are close to each other, and the model BBBAV14 has the lowest maximal value. For the models BBBAV14, BBBParis, and DH, respectively, the disc radiates its maximal flux at larger radii than the APR EOS. Both the model RMF soft and quark-star model Q still belong to this first group, and they have accretion discs with an inner edge $r / M \simeq 5.4$. Nevertheless, the flux profiles of their disc radiation are different. The highest values for $F(r)$ among all the models of the first group are obtained for the RMF soft model. The flux emitted by the accretion disc around the quark star described by the bag model equation of state $\mathrm{Q}$ is lower by a factor of around 0.7 , compared to the fluxes emitted by discs around neutron stars, but the flux profile is very similar to the DH model profile. The only difference between them is in the location of the inner edge of the disc. The quark-star model CFL150 also belongs to the first group. The inner disc edge is located at $r / M \simeq 5.6$ for this type of EOS, but the maximal flux emitted by the disc is close to the one emitted by the BBBParis model.

The RMF stiff and STOS-type neutron-star models form a second group, since the accretion discs around them emit a flux that is far lower than the fluxes of the first group. The stellar radii of these stars are much greater than those of the stars in the first group, and their marginally stable orbits are located at even larger radii, between $r / M \simeq 7$ and 11.7 , respectively. In the STOS-type models, the maximum values of their fluxes increase, and the radii of the left boundary of $F(r)$ decrease, with increasing star temperatures. With the smallest radius of the inner edge, the EOS RMF stiff gives the highest maximal flux in the second group. The temperature distribution in the disc, shown in Fig. 3, follows the same pattern as the one described for the flux profiles.

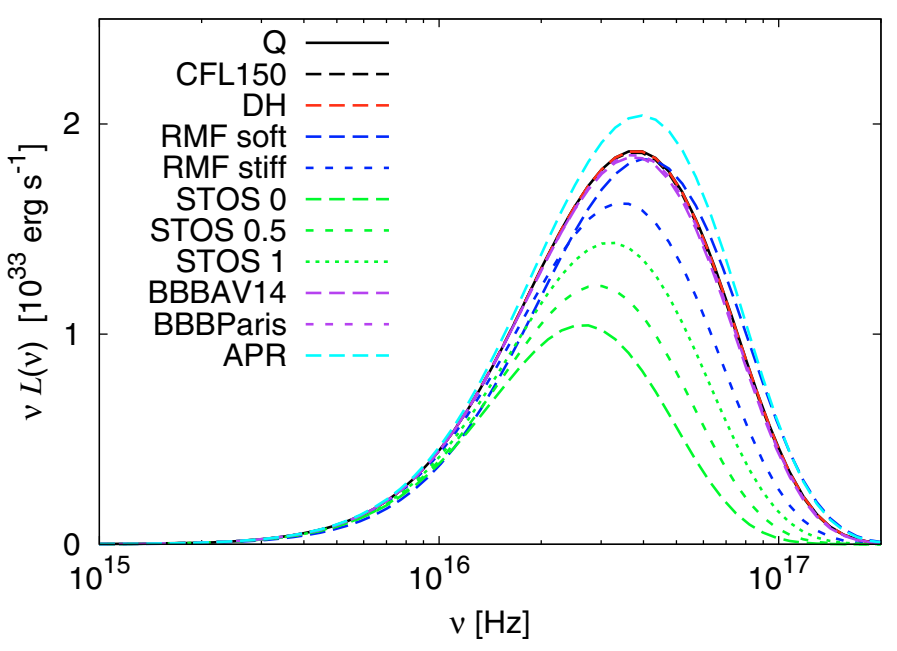

Fig. 4. The emission spectrum of the accretion disc around rotating neutron and quark stars with the same total mass $M=1.8 M_{\odot}$, and rotational velocity $\Omega=5 \times 10^{3} \mathrm{~s}^{-1}$.

In Fig. 4, we present emission spectra of the discs, which in a similar way to the flux and temperature curves, also exhibit some distinctive features. Both the amplitude and cut-off frequency of the spectra are determined roughly by the maximum values of $F(r)$ : for higher maximal flux values of an accretion disc around a given stellar model, we obtain higher maximal amplitudes of the disc spectra, and they are shifted to higher frequencies. This is clearly seen in Fig. 4 for the neutron stars with the RMF stiff and the STOS type EOS's. However, there are some exceptions. The spectral maximum is lower for the RMF soft model than the APR type EOS, but its cut-off is still located at a higher frequency. The spectral maxima are almost the same for the BBBAV14 and BBBParis models, but the cutoff frequency is higher for the BBBAV14 type EOS. The spectral maxima for all the models in the plot are located between $2 \times 10^{16}$ and $4 \times 10^{16} \mathrm{~Hz}$. The spectral features of the neutron-star model DH and of both types of quark-star models are essentially the same, and thus only the morphological properties of the accretion disc, i.e., the radial distribution of the flux or the disc temperature, can be used to differentiate between these types of compact stellar objects.

\subsection{Accretion discs around neutron and quark stars rotating with Keplerian velocities}

As a second case of comparison between the neutron and quark star properties, we consider the Keplerian models of the neutron and quark stars, where they rotate at the maximal frequency allowed by their physical properties. We fix the central density for all the stellar models to $\rho_{\mathrm{c}}=10^{15} \mathrm{~g} / \mathrm{cm}^{3}$, and we compute the physical parameters of all the stellar models for the case of Keplerian rotation. The results are presented in Table 2.

The flux curves for these configurations are shown in Fig. 5. Here each EOS type has very distinctive features. Only the STOS-type stars, and the models BBBAV14 and BBBParis produce similar flux profiles. Nevertheless, there is a temperature dependence for the models STOS in the location of the innerdisc edge $(r / M=4.1-4.2)$, and of the maximal value of the photon flux emerging from the disc: with increasing temperatures, we obtain greater radii for the inner edge of the disc, and the maximal flux values are inversely proportional to the temperature of the star. For the models BBBAV14 and BBBParis, 
Table 2. Physical parameters of the neutron and quark stars rotating at Keplerian frequencies. All stars have the same central density $\rho_{\mathrm{c}}$.

\begin{tabular}{llllllllllll}
\hline \hline EOS & DH & RMF soft & RMF stiff & STOS 0 & STOS 0.5 & STOS 1 & BBBAV14 & BBBParis & APR & $Q$ & CFL300 \\
\hline$\rho_{\mathrm{c}}\left[10^{15} \mathrm{~g} / \mathrm{cm}^{3}\right]$ & 1.00 & 1.00 & 1.00 & 1.00 & 1.00 & 1.00 & 1.00 & 1.00 & 1.00 & 1.00 & 1.00 \\
$M\left[M_{\odot}\right]$ & 1.78 & 1.84 & 2.78 & 3.49 & 3.42 & 3.35 & 1.53 & 1.54 & 2.10 & 2.79 & 4.76 \\
$M_{0}\left[M_{\odot}\right]$ & 1.98 & 2.03 & 3.23 & 4.15 & 4.03 & 3.92 & 1.68 & 1.70 & 2.40 & 3.29 & 5.98 \\
$R_{\mathrm{e}}[\mathrm{km}]$ & 16.24 & 17.78 & 18.42 & 19.15 & 19.24 & 19.43 & 15.58 & 15.74 & 15.52 & 17.07 & 20.50 \\
$\Omega\left[10^{3} \mathrm{~s}^{-1}\right]$ & 7.44 & 6.91 & 7.74 & 7.99 & 7.81 & 7.60 & 7.37 & 7.29 & 8.96 & 9.07 & 8.59 \\
$\Omega_{\mathrm{p}}\left[10^{4} \mathrm{~s}^{-1}\right]$ & 7.44 & 6.91 & 7.74 & 7.99 & 7.81 & 7.60 & 7.37 & 7.29 & 8.97 & 9.08 & 8.79 \\
$T / W\left[10^{-1}\right]$ & 1.14 & 1.62 & 1.54 & 1.48 & 1.38 & 1.27 & 1.12 & 1.12 & 1.95 & 2.27 & 2.18 \\
$c J / G M_{\odot}^{2}$ & 2.15 & 2.91 & 5.94 & 8.98 & 8.31 & 7.66 & 1.61 & 1.64 & 3.83 & 7.15 & 1.96 \\
$I\left[10^{45} \mathrm{~g} \mathrm{~cm}^{2}\right]$ & 2.54 & 3.70 & 6.74 & 9.88 & 9.34 & 8.86 & 1.92 & 1.97 & 3.75 & 6.91 & 2.01 \\
$\Phi_{2}\left[10^{44} \mathrm{~g} \mathrm{~cm}^{2}\right]$ & 34.02 & 73.57 & 99.54 & 124.77 & 110.35 & 97.48 & 27.12 & 27.84 & 69.85 & 13.15 & 33.29 \\
$h_{+}[\mathrm{km}]$ & 0.00 & 0.00 & -3.59 & 1.65 & 1.37 & 1.06 & 0.00 & 0.00 & -3.14 & -3.14 & -2.83 \\
$h_{-}[\mathrm{km}]$ & 6.94558 & -3.91 & 17.75 & 24.54 & 23.11 & 21.64 & 4.89 & 4.91 & 13.63 & 0.13 & -0.08 \\
$\omega_{\mathrm{c}} / \Omega\left[10^{-1}\right]$ & 5.44 & 5.15 & 6.90 & 7.95 & 7.88 & 7.82 & 4.94 & 4.95 & 6.07 & 6.96 & 8.82 \\
$r_{\mathrm{e}}[\mathrm{km}]$ & 13.37 & 14.72 & 13.66 & 13.05 & 13.35 & 13.72 & 13.13 & 13.27 & 11.91 & 11.99 & 11.06 \\
$r_{\mathrm{p}} / r_{\mathrm{e}}$ & 0.55 & 0.49 & 0.52 & 0.54 & 0.55 & 0.56 & 0.55 & 0.55 & 0.47 & 0.44 & 0.51 \\
\hline
\end{tabular}

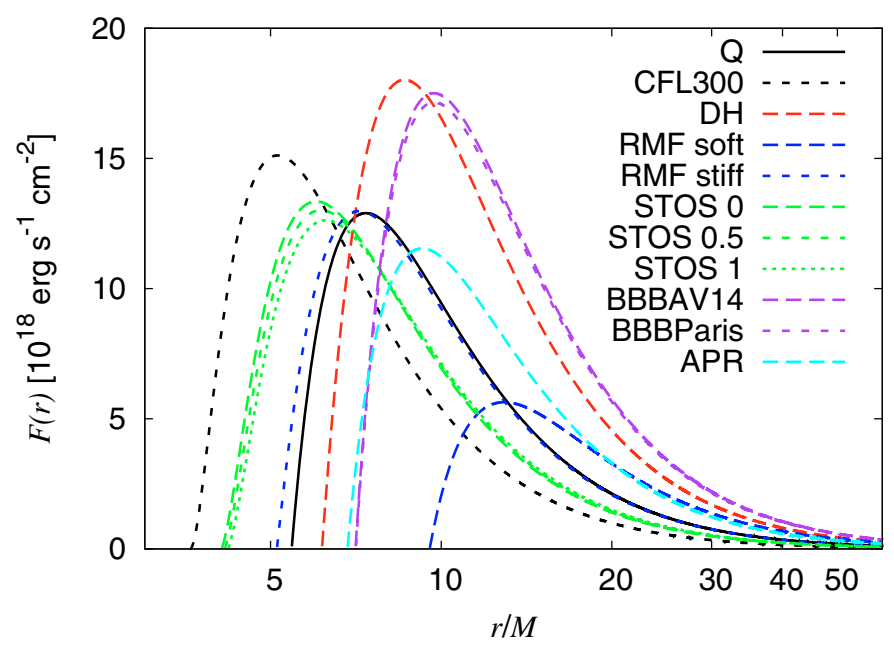

Fig. 5. The time-averaged flux radiated by a thin accretion disc around neutron and quark stars rotating at the Keplerian velocity. All the stars have the same central density $\rho_{\mathrm{c}}=10^{15} \mathrm{~g} / \mathrm{cm}^{3}$.

we see that the location of the inner edge of the accretion discs around these types of neutron stars are essentially the same $(r / M \simeq 7.1)$, but the model BBBAV14 produces a higher maximal flux than the BBBPAris type EOS does. Although the thin disc rotating around the APR type star has an inner edge at only a slightly smaller radius than the discs for the models BBBAV14 and BBBParis, respectively, its maximal radiated-away flux is a factor of 1.4 lower than those of neutron stars with the latter types of EOS.

The inner edge of the accretion disc around the quark star CFL300 is located at the smallest radius $(r / M \simeq 3.6)$, compared with the other EOS models in this configuration, whereas the model RMF soft has the greatest radius for the inner edge $(r / M \simeq 9.5)$. The flux values for the RMF soft type star are also the smallest ones. The maximal flux of the model DH is the greatest in this group, and it is at least a factor of 3.5 greater than the one for the model RMS stiff. The flux profiles of the $\mathrm{Q}$ type quark star and of the neutron star with the RMF stiff type EOS are very similar, but the inner edge of the disc rotating around the neutron star is located at a lower radius $(r / M \simeq 5.5$ for the quark star, and $r / M \simeq 5.1$ for the neutron star). For the radial

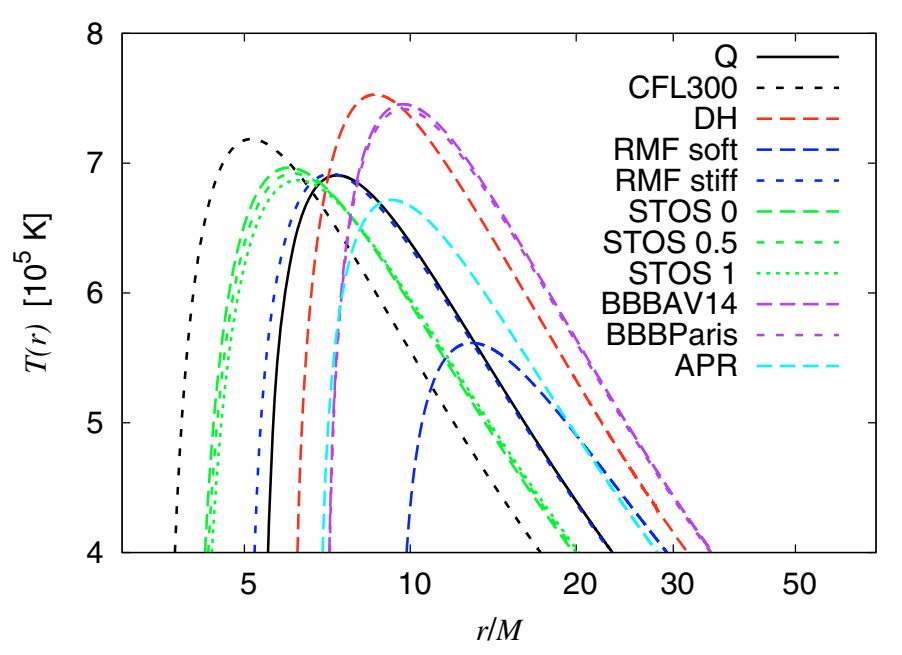

Fig. 6. The temperature distribution by a thin accretion disc around neutron and quark stars rotating at the Keplerian velocity. All the stars have the same central density $\rho_{\mathrm{c}}=10^{15} \mathrm{~g} / \mathrm{cm}^{3}$.

distributions of the disc temperature, we obtain the same characteristic behavior and groups of models, which we present in Fig. 6.

The spectral features, as seen in Fig. 7, are strongly influenced by the flux and temperature distributions of the disc. For the case of Keplerian rotation, the emission spectra of the disc are rather different from those of the previous configuration, of similar gravitational mass and angular velocity. The proportionality between the location of the inner-disc edge and the maximal amplitudes of the spectra holds for this case as well. Therefore, the greatest spectral maximum is produced by the quark-star model CFL300, whereas the smallest one is obtained for the RMF stiff model. The STOS models also have high amplitudes in their spectra, and their maximal values, in contrast with the previous case, are inversely proportional to the temperature.

There are no large differences in the cut-off frequencies in the spectra, which are around $2 \times 10^{16}-3 \times 10^{16} \mathrm{~Hz}$. The spectrum belonging to the RMF stiff type EOS has its maximum at the lowest frequency, and the spectral maxima for the models $\mathrm{DH}$, BBBAV14, and BBBParis are located at the highest frequencies. Since the spectra for the latter two models are very similar, we 


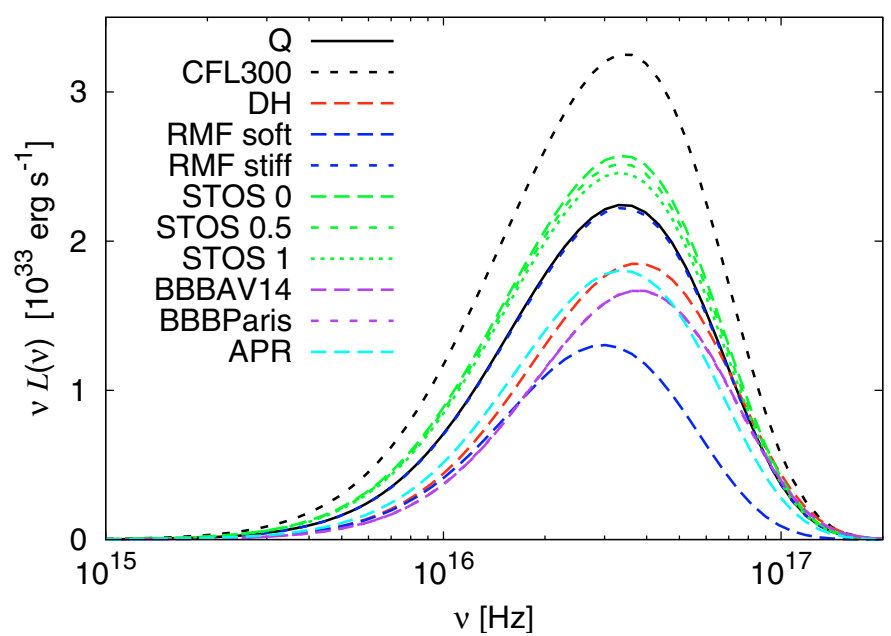

Fig. 7. The emission spectrum of the accretion disc around neutron and quark stars rotating at the Keplerian velocity. All the stars have the same central density $\rho_{\mathrm{c}}=10^{15} \mathrm{~g} / \mathrm{cm}^{3}$.

can only use the differences in the flux maxima to discriminate between them. This is also the case for the quark-star model Q and the RMF-stiff-type neutron star as well, where the only discriminating factor is the difference in the locations of the inner disc edges. The models APR and DH also have similar spectra, but both their maxima and cut-off frequencies exhibit some differences.

\subsection{Accretion discs onto neutron and quark stars of fixed central density and eccentricity}

As a third example of the comparison between the radiation properties of the thin accretion discs around neutron and quark stars, we consider the case in which the rotating central compact objects have the same central density and eccentricity $r_{\mathrm{p}} / r_{\mathrm{e}}$, where $r_{\mathrm{p}}$ is the polar radius and $r_{\mathrm{e}}$ is the equatorial radius of the star. The basic fundamental parameters of the rotating neutron stars and quark stars are presented in Table 3.

The physical parameters of the rotating stars were obtained for all the considered equations of state by choosing a central density $\rho_{\mathrm{c}}=1 \times 10^{15} \mathrm{erg} / \mathrm{cm}^{3}$, and an eccentricity $r_{\mathrm{p}} / r_{\mathrm{e}}=0.45$. In Fig. 8 we present the radial distribution of the energy flux $F(r)$ in this stellar configuration for the neutron star models DH, RMF soft/stiff, STOS $(T=0.0, T=0.5 \mathrm{MeV}$, and $T=1.0 \mathrm{MeV})$, BBBAV14, BBBParis, and APR, and for the quark-star models $\mathrm{Q}$ and CFL300.

Figure 8 illustrates that different equations of state with the same central density and eccentricity produce rather different geometries around the compact central object and, in turn, determine rather distinct emissivity properties for the accretion discs. The distribution of the radial flux profiles is similar to the Keplerian case, shown in Fig. 5, but with some significant differences. The differences between the amplitudes of the disc radiation for the central objects with the same $\rho_{\mathrm{c}}$ and $r_{\mathrm{e}} / r_{\mathrm{p}}$ ratio are smaller than those evident in Fig. 5. This is caused by the considerable lower maximal flux values for the models DH, BBBVA14 and BBBParis, respectively, compared to the maxima reached in the Keplerian case. The other EOS types produce more or less the same, or somewhat higher, flux maxima than in the case of Keplerian rotation. By considering the inner edges of the disc, we find that the radial distribution of their location is very similar to the one seen in Fig. 5. Nonetheless, the differences between

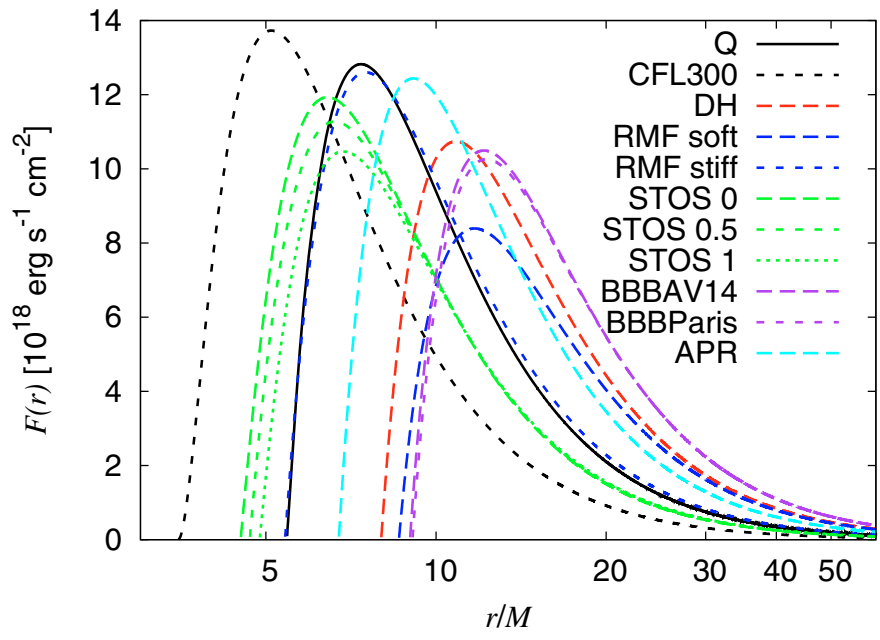

Fig. 8. The time-averaged flux radiated by a thin accretion disc around rotating neutron and quark stars, with the same central density $\left(\rho_{\mathrm{c}}=\right.$ $\left.10^{15} \mathrm{~g} / \mathrm{cm}^{3}\right)$ and eccentricity $\left(r_{\mathrm{p}} / r_{\mathrm{e}}=0.45\right)$.

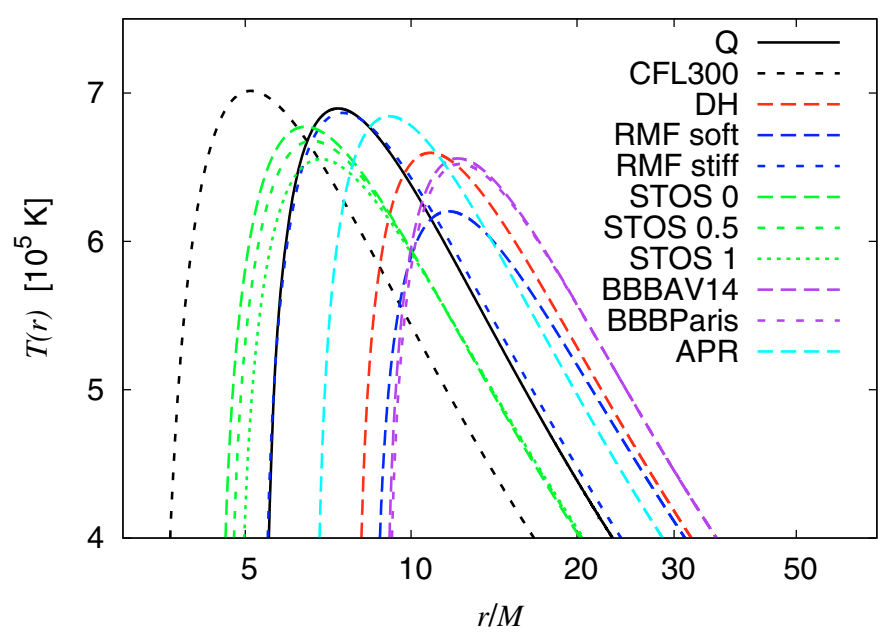

Fig. 9. Temperature distribution in a thin accretion disc around rotating neutron and quark stars, with the same central density $\left(\rho_{\mathrm{c}}=10^{15} \mathrm{~g} / \mathrm{cm}^{3}\right)$ and eccentricity $\left(r_{\mathrm{p}} / r_{\mathrm{e}}=0.45\right)$.

the flux profiles for the STOS models are bigger, and there is also a shift in the inner edge of the disc to higher radii for the models DH, BBBVA14 and BBBParis, which determines a significant reduction in their flux values. These features can also be seen in Fig. 9, where we display the temperature distribution of the disc surface in thermodynamical equilibrium.

Important differences are also present in the disc spectra. The black-body spectrum of the equilibrium thermal radiation emitted from the accretion disc for each type of star is shown in Fig. 10. The quark stars together with the STOS-type neutronstars produce greater maximal amplitudes in the disc emission spectra than the other neutron stars in this group. The inverse proportionality between the temperature of the star and the maximal amplitudes of the spectra holds again for the STOS models, similarly to the Keplerian case. However, the spectrum of the quark star for the EOS Q and the spectrum of the neutron star with the RMF-stiff-type EOS are decoupled in this case, and the disc spectrum for the quark star is more similar to the spectrum of the model STOS 1 . The latter has a slightly lower cut-off frequency, and these two types of EOS could be discriminated from each other by comparing the radial distributions of both the flux 
Table 3. Physical parameters of the rapidly rotating compact stars with same central density and eccentricity.

\begin{tabular}{|c|c|c|c|c|c|c|c|c|c|c|c|}
\hline EOS & $\mathrm{DH}$ & RMF soft & RMF stiff & STOS 0 & STOS 0.5 & STOS 1 & BBBAV14 & BBBParis & APR & $Q$ & CFL300 \\
\hline$\rho_{\mathrm{c}}\left[10^{15} \mathrm{~g} / \mathrm{cm}^{3}\right]$ & 1.00 & 1.00 & 1.00 & 1.00 & 1.00 & 1.00 & 1.00 & 1.00 & 1.00 & 1.00 & 1.00 \\
\hline$M\left[M_{\odot}\right]$ & 1.69 & 1.72 & 2.62 & 3.32 & 3.27 & 3.22 & 1.45 & 1.46 & 2.05 & 2.80 & 4.50 \\
\hline$M_{0}\left[M_{\odot}\right]$ & 1.88 & 1.91 & 3.04 & 3.95 & 3.86 & 3.77 & 1.59 & 1.60 & 2.35 & 3.30 & 5.67 \\
\hline$R_{\mathrm{e}}[\mathrm{km}]$ & 20.01 & 19.47 & 20.95 & 22.17 & 22.66 & 23.24 & 19.32 & 19.51 & 16.14 & 16.95 & 21.59 \\
\hline$\Omega\left[10^{3} \mathrm{~s}^{-1}\right]$ & 6.82 & 6.71 & 7.34 & 7.49 & 7.28 & 7.05 & 6.74 & 6.67 & 8.91 & 9.07 & 8.36 \\
\hline$\Omega_{\mathrm{p}}\left[10^{3} \mathrm{~s}^{-1}\right]$ & 5.26 & 5.63 & 6.06 & 6.18 & 5.93 & 5.67 & 5.15 & 5.09 & 8.19 & 9.25 & 7.50 \\
\hline$T / W\left[10^{-2}\right]$ & 9.14 & 13.77 & 12.75 & 12.26 & 11.37 & 10.45 & 8.91 & 8.89 & 18.64 & 22.87 & 19.27 \\
\hline$c J / G M_{\odot}^{2}$ & 1.72 & 2.33 & 4.75 & 7.39 & 6.90 & 6.41 & 1.28 & 1.30 & 3.58 & 7.22 & 16.55 \\
\hline$I\left[10^{45} \mathrm{~g} \mathrm{~cm}^{2}\right]$ & 2.22 & 3.06 & 5.68 & 8.66 & 8.32 & 7.99 & 1.67 & 1.71 & 3.53 & 6.99 & 1.73 \\
\hline$\Phi_{2}\left[10^{44} \mathrm{~g} \mathrm{~cm}^{2}\right]$ & 25.00 & 53.68 & 71.23 & 92.12 & 82.80 & 74.09 & 19.86 & 20.39 & 63.51 & 13.34 & 2.55 \\
\hline$h_{+}[\mathrm{km}]$ & 0.00 & 0.00 & 0.00 & 0.00 & 0.00 & 0.00 & 0.00 & 0.00 & 0.00 & -3.09 & 1.63 \\
\hline$h_{-}[\mathrm{km}]$ & 1.28 & 5.25 & 12.07 & 18.34 & 16.85 & 15.26 & 0.00 & 0.00 & 12.15 & -0.34 & 35.70 \\
\hline$\omega_{\mathrm{c}} / \Omega\left[10^{-1}\right]$ & 5.25 & 4.98 & 6.71 & 7.79 & 7.74 & 7.68 & 4.75 & 4.76 & 6.01 & 6.97 & 8.69 \\
\hline$r_{\mathrm{e}}[\mathrm{km}]$ & 17.37 & 16.71 & 16.70 & 16.69 & 17.31 & 18.01 & 17.07 & 17.25 & 12.69 & 13.41 & 8.75 \\
\hline$r_{\mathrm{p}} / r_{\mathrm{e}}$ & 0.45 & 0.45 & 0.45 & 0.45 & 0.45 & 0.45 & 0.45 & 0.45 & 0.45 & 0.45 & 0.45 \\
\hline
\end{tabular}

Table 4. The radius of the inner disc edge $r_{\text {in }}$ and the efficiency $\epsilon$.

\begin{tabular}{|c|c|c|c|c|c|c|c|c|c|c|c|}
\hline$\overline{\mathrm{EOS}}$ & $\overline{\mathrm{DH}}$ & $\overline{\text { RMF soft }}$ & $\overline{\text { RMF stiff }}$ & $\overline{\text { STOS 0 }}$ & $\overline{\text { STOS } 0.5}$ & STOS 1 & $\overline{\text { BBBAV14 }}$ & BBBParis & APR & $Q$ & CFL300 \\
\hline (a) $r_{\text {in }}[\mathrm{km}]$ & 13.88 & 12.10 & 18.81 & 32.18 & 26.33 & 22.11 & 13.83 & 13.76 & 13.85 & 14.24 & 14.84 \\
\hline$\epsilon$ & 0.0679 & 0.0667 & 0.0589 & 0.0400 & 0.0463 & 0.0527 & 0.0673 & 0.0682 & 0.0742 & 0.0685 & 0.0683 \\
\hline (b) $r_{\text {in }}[\mathrm{km}]$ & 16.24 & 25.97 & 21.13 & 21.17 & 21.00 & 20.87 & 16.02 & 16.17 & 21.22 & 22.51 & 23.83 \\
\hline$\epsilon$ & 0.0676 & 0.0488 & 0.0802 & 0.0929 & 0.0913 & 0.0894 & 0.0611 & 0.0610 & 0.0663 & 0.0814 & 0.1172 \\
\hline (c) $r_{\text {in }}[\mathrm{km}]$ & 20.01 & 21.95 & 20.96 & 22.18 & 22.66 & 23.24 & 19.32 & 19.52 & 20.45 & 22.61 & 23.26 \\
\hline$\epsilon$ & 0.0557 & 0.0528 & 0.0768 & 0.0869 & 0.0845 & 0.0819 & 0.0503 & 0.0502 & 0.0666 & 0.0814 & 0.1097 \\
\hline
\end{tabular}

and temperature of their disc. The spectra of the group consisting of the models DH, RMF soft, BBBAV14, and BBBParis are similar. The spectra indeed coincide for the latter two models. For this case the different spatial distributions of the radiating disc can help again in the discrimination. There is no such a problem with the APR type EOS, since it produces higher spectral amplitudes than the other cases. The maxima of all the spectra are located in a very narrow frequency range between $2 \times 10^{16}$ and $2.2 \times 10^{16} \mathrm{~Hz}$, although the numerical values of the flux maxima are rather different, more or less following those of the flux maxima.

\section{Discussions and final remarks}

We have considered the basic physical properties of matter forming a thin accretion disc around rapidly rotating neutron and quark stars. The physical parameters of the disc-effective potential, flux, and emission spectrum profiles-have been obtained explicitly for several equations of state of the neutron matter and for two types of quark stars. All the astrophysical quantities related to the observable properties of the accretion disc, can be obtained from the metric of the central compact object. Because of their differences in the space-time structure, the quark stars exhibit some very important differences in terms of the disc properties, compared to neutron stars. This procedure allows us not only to discriminate between neutron and quark stars, but also indicates some distinct signatures that can be used to differentiate between neutron stars described by different equations of state, and distinguish between the different phases of the quark matter.

Another observable physical parameter of the disc that could help in distinguishing between different classes of compact objects is the efficiency $\epsilon$ of the conversion of the accreting mass into radiation, given by Eqs. (17) and (18). The values of $\epsilon$

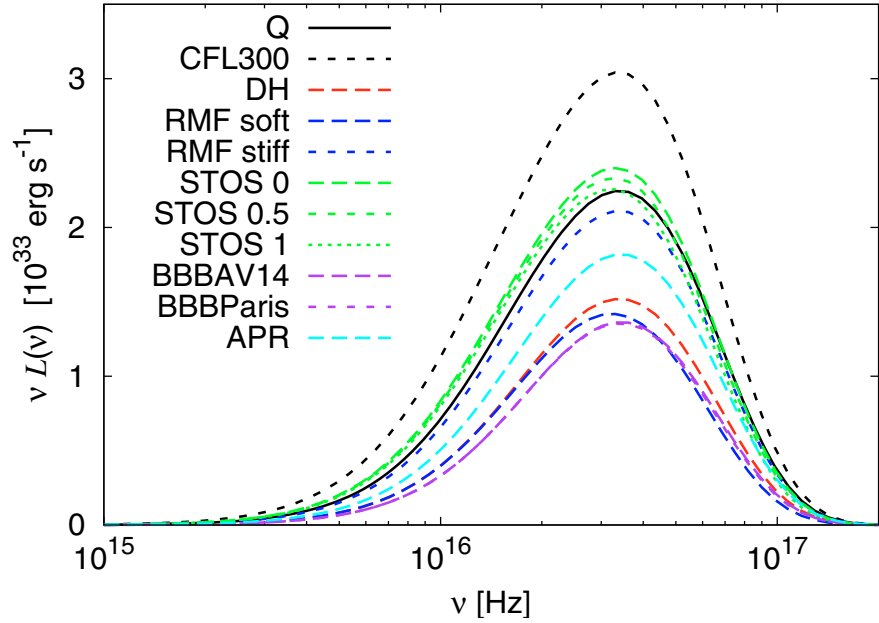

Fig. 10. The emission spectrum of the accretion disc around rotating neutron and quark stars, with the same central density $\left(\rho_{\mathrm{c}}=10^{15} \mathrm{~g} / \mathrm{cm}^{3}\right)$ and eccentricity $\left(r_{\mathrm{p}} / r_{\mathrm{e}}=0.45\right)$.

measure the efficiency of the energy generating mechanism of the mass accretion. The amount of energy released by the matter leaving the marginally stable orbit, or the inner edge of the disc touching the surface of the star, and being transferred to the star, is the binding energy $\widetilde{E}_{\mathrm{ms}}$ or $\widetilde{E}_{\mathrm{e}}$. In Table 4 we present the efficiency of the energy conversion for the different equations of state considered in the present paper. The case of stars of similar total mass and angular velocity is represented in (a), stars in Keplerian rotation are described in (b), while the case of the stars with the same central density and eccentricity is shown in (c).

For rotating compact stars of similar total mass and angular velocity the values of $\epsilon$ are in the range $4 \%$ and $8 \%$, indicating 
that in this case there is no significant difference in the efficiency of the mass-to-radiation conversation mechanism. The least efficient conversion mechanism is provided by the STOS models, especially at the zero temperature of the star, whereas the APR-type EOS provides the most efficient conversion mechanism with $\epsilon=7.4 \%$. The quark stars with $\epsilon=6.8 \%$ are also efficient engines for converting the accreted mass into outgoing radiation.

The values of $\epsilon$ for the compact general relativistic objects in Keplerian rotation are given in the second two lines of Table 4.

In this case, the quark star model CFL300 provides the most efficient mechanism for the conversion, with $\epsilon=11.7 \%$. The quark stars described by the EOS Q have also a high conversion efficiency, of $\epsilon=8.15 \%$. The conversion efficiency for the models STOS are much higher than those in the previous stellar configuration. Their efficiencies can reach for this case a value of $\epsilon=9 \%$. The other models have efficiencies on the order of $5-6 \%$.

The last two lines in Table 4 contains the values of the efficiency for stellar models with the same eccentricity and central density, respectively. The range of the values of $\epsilon$ is similar to the previous case, although in general the numerical values are somewhat lower. We obtain again the highest efficiency for the model CFL300, and the smallest ones for the neutron star models DH, RMF soft, BBBAV14, and BBBParis, with $\epsilon$ about $5 \%$. The STOS-type neutron stars and the Q-type quark stars have higher efficiencies $\epsilon=7-8 \%$.

As shown by the flux integral in Eq. (6), and the explicit expressions of the specific energy, specific angular momentum, and angular velocity given by Eqs. (11)-(13), the different characteristics of the radial flux distribution over the accretion disc, the disc spectra, and the conversion efficiency are caused by differences between the metric potentials of the neutron and quark stars. Even if the total mass and angular velocity are the same for each type of rotating central object, producing similar values of $\Omega, \widetilde{E}$, and $\widetilde{L}$, the radiation properties of the accretion discs around these objects exhibit discernible differences. The reason is that the proper volume, and in turn the function $\sqrt{-g}$, used in the calculation of the flux integral, are highly dependent on the behavior of the metric component $g_{r r}=(\partial \bar{r} / \partial r)^{2} g_{\overline{r r}}$. The last quantity is dependent on the $r$-derivatives of the metric functions $\rho(r)$ and $\gamma(r)$, by means of the coordinate transformation between $\bar{r}$ and $r$, which are extremely sensitive to the slope of $\rho(r)$ and $\gamma(r)$. As a result, although the inner edges of the discs are located at almost the same radii, the amplitudes of the energy fluxes emerging form the disc surface and propagating in any solid angle may have considerable differences for different equations of state. These features also provide distinctive features in the disc spectra for the various types of central stars.

In conclusion, the observational study of the thin accretion discs around rapidly rotating compact objects can provide a powerful tool for distinguishing between standard neutron stars and stars with exotic equations of state, which have undergone, for example, a phase transition from the neutron phase to a quark phase, as well as for discriminating between the different equations of state of dense matter.

Acknowledgements. We would like to thank to the anonymous referee for suggestions and comments that helped us to significantly improve the manuscript. Z.K. is indebted to the colleagues in the Department of Physics and Center for Theoretical and Computational Physics of the University of Hong Kong for their support and warm hospitality. K.S.C. is supported by the GRF grant number HKU 7013/06P of the government of the Hong Kong SAR. The work of T.H. is supported by the GRF grant number HKU 702507 of the Government of the Hong Kong SAR.

\section{References}

Akmal, A., Pandharipande, V. R., \& Ravenhall, D. G. 1998, Phys. Rev. C, 58, 1804

Alcock, C., Farhi, E., \& Olinto, A. 1986, ApJ, 310, 261

Alford, M. G., Rajagopal, K., \& Wilczek, F. 1999, Nucl. Phys. B, 537, 433

Alford, M. G., Rajagopal, K., Schaefer, T., \& Schmitt, A. 2008, Rev. Mod. Phys., 80,1455

Baldo, M., Bombaci, I., \& Burgio, G. F. 1997, A\&A, 328, 274

Baym, G., Bethe, H. A., \& Pethick, C. J. 1971a, Nucl. Phys. A, 175, 225

Baym, G., Pethick, C. J., \& Sutherland, P. 1971b, ApJ, 170, 299

Bhattacharyya, S. 2002, A\&A, 383, 524

Bhattacharyya, S., Thampan, A. V., Misra, R., \& Datta, B. 2000, ApJ, 542, 473

Bhattacharyya, S., Thampan, A. V., \& Bombaci, I. 2001a, A\&A, 372, 925

Bhattacharyya, S., Misra, R., \& Thampan, A. V. 2001b, ApJ, 550, 841

Bhattacharyya, S., Bhattacharya, D., \& Thampan, A. V. 2001c, MNRAS, 325, 989

Bodmer A. R. 1971, Phys. Rev. D, 4, 1601

Broderick, A. E., \& Narayan, R. 2006, ApJ, 638, L21

Chan, T. C., Cheng, K. S., Harko, T., et al. 2009, ApJ, in press, [arXiv:0902.0653]

Cheng, K. S., \& Dai, Z. G. 1996, Phys. Rev. Lett., 77, 1210

Cheng, K. S., \& Dai, Z. G. 1998, Phys. Rev. Lett., 80, 18

Cheng, K. S., \& Harko, T. 2001, Phys. Rev. D, 62, 083001

Cheng, K. S., \& Harko, T. 2003, ApJ, 596, 451

Cheng, K. S., Dai, Z. G., \& Lu, T. 1998a, Int. J. Mod. Phys. D, 7, 139

Cheng, K. S., Dai, Z. G., Wei, D. M., \& Lu, T. 1998b, Science, 280, 407

Dai, Z. G., Peng, Q. H., \& Lu, T. 1995, ApJ, 440, 815

Dey, M., Bombacci, I., Dey, J., Ray, S., \& Samanta, B. C. 1998, Phys. Lett. B, 438,123

Douchin, F., \& Haensel, P. 2001, A\&A, 380, 151

Feynman, R. P., Metropolis, N., \& Teller, E. 1949, Phys. Rev., 75, 1561

Gondek-Rosinska, D., Bulik, T., Zdunik, L., et al. 2000, A\&A, 363, 1005

Gourgoulhon, E., Haensel, P., Livine, R., et al. 1999, A\&A, 349, 851

Guzman, F. S. 2006, Phys. Rev. D, 73, 021501

Haensel, P., Zdunik, J. L., \& Schaeffer, R. 1986, A\&A, 160, 121

Harko, T., \& Cheng, K. S. 2002, A\&A, 385, 94

Harko, T., \& Cheng, K. S. 2005, ApJ, 622, 1033

Harko, T., \& Cheng, K. S. 2006, ApJ, 643, 318

Harko, T., Kovács, Z., \& Lobo, F. S. N. 2008, Phys. Rev. D, 78, 084005

Harko, T., Kovács, Z., \& Lobo, F. S. N. 2009, Phys. Rev. D, 79, 064001

Horvath, J. E., \& Lugones, G. 2004, A\&A, 422, L1

Itoh, N. 1970, Prog. Theor. Phys., 44, 291

Kubis, S., \& Kutschera, M. 1997, Phys. Lett. B, 399, 191

Lugones, G., \& Horvath, J. E. 2002, Phys. Rev. D, 66, 074017

Luminet, J. P. 1979, A\&A, 75, 228

Madsen, J. 2000, Phys. Rev. Lett., 85, 10

Novikov, I. D., \& Thorne, K. S. 1973, in Black Holes, ed. C. DeWitt, \& B. DeWitt (New York: Gordon and Breach)

Nozawa, T., Stergioulas, N., Gourgoulhon, E., \& Eriguchi, Y. 1998, A\&A, 132, 431

Page, D. N., \& Thorne, K. S. 1974, ApJ, 191, 499

Page, D., \& Usov, V. V. 2002, Phys. Rev. Lett., 89, 131101

Pandharipande, V. R. 1971, Nucl. Phys. A, 178, 123

Pun, C. S. J., Kovács, Z., \& Harko, T. 2008a, Phys. Rev. D, 78, 024043

Pun, C. S. J., Kovács, Z., \& Harko, T. 2008b, Phys. Rev. D, 78, 084015

Rapp, R., Schaffer, T., Shuryak, E. V., \& Velkovsky, M. 2000, Ann. Phys. (N. Y.), 280, 35

Shakura, N. I., \& Sunyaev, R. A. 1973, A\&A, 24, 33

Shen, H., Toki, H., Oyamatsu, K., \& Sumiyoshi, K. 1998, Nucl. Phys. A, 637, 435

Shibata, M., \& Sasaki, M. 1998, Phys. Rev. D, 58, 104011

Sibgatullin, N. R., \& Sunyaev, R. A. 1998, Astron. Lett., 24, 774

Stergioulas, N. 2003, Living Rev. Rel., 6, 3

Stergioulas, N., \& Friedman, J. L. 1995, ApJ, 444, 306

Stergioulas, N., Kluzniak, W., \& Bulik, T. 1999, A\&A, 352, L116

Thampan, A. V., \& Datta, B. 1998, MNRAS, 297, 570

Thorne, K. S. 1974, ApJ, 191, 507

Torres, D. 2002, Nucl. Phys. B, 626, 377

Urry, C. M., \& Padovani, P. 1995, Publ. Astron. Soc. Pacific, 107, 803

Usov, V. V. 1998a, Phys. Rev. Lett., 80, 230

Usov, V. V. 1998b, Phys. Rev. Lett., 81, 4775

Yuan, Y. F., Narayan, R., \& Rees, M. J. 2004, ApJ, 606, 1112

Witten, E. 1984, Phys. Rev. D, 30, 272

Zdunik, J. L. 2000, A\&A, 359, 311 